\title{
Effects of group composition and level of selection in the evolution of cooperation in artificial ants
}

\author{
Andres Perez-Uribe ${ }^{1}$, Dario Floreano ${ }^{1}$, and Laurent Keller ${ }^{2}$ \\ (1) Autonomous Systems Lab., Swiss Federal Institute of Technology-Lausanne, \\ Switzerland, http://asl.epfl.ch \\ (2) Institute of Ecology, Laboratory for Zoology, University of Lausanne, Switzerland, \\ http://www.unil.ch/izea
}

\begin{abstract}
Since ants and other social insects have long generation time, it is very difficult for biologists to study the origin of complex social organization by guided evolution (a process where the evolution of a trait can be followed during experimental evolution). Here we use colonies of artificial ants implemented as small mobile robots with simple vision and communication abilities to explore these issues. In this paper, we present results concerning the role of relatedness (genetic similarity) and levels of selection (individual and colony-level selection) on the evolution of cooperation and division of labor in simulated ant colonies. In order to ensure thorough statistical analysis, the evolutionary experiments, herein reported, have been carried out using "minimalist" simulations of the collective robotics evolutionary setup. The results show that altruistic behaviors have low probability of emerging in heterogeneous colonies evolving under individual-level selection and that colonies with high genetic relatedness display better performance.
\end{abstract}

Keywords: Evolution, cooperation, division of labor, altruism, social insects.

\section{Introduction}

It has been estimated that ants compose about $15 \%$ of the animal biomass in most terrestrial environments, and up to one-third of the animal biomass of the Amazon rain forest consists of ants and termites [1]. Their success might come from the fact that social interactions can compensate for individual limitations, both in terms of physical and cognitive capabilities. Indeed, herds and packs allow animals to attack larger prey and increase their chances for survival and mating [2], while organizations and teams facilitate information sharing and problem solving. The complexity of any society results from the local interactions among its members. Synthesizing and analyzing coherent collective behavior from individual interactions is one of great challenges in both ethology and artificial intelligence.

Social insects not only exhibit highly organized collective behaviors. They provide some of the most remarkable examples of altruistic behavior with their 
worker caste, whose individuals forego their own reproduction to enhance reproduction of the queen. These and other examples of group harmony and cooperation have given rise to the concept that colonies are harmonious fortress-factories in which individual-level selection is muted, with the result that colony-level selection reigns. In other words, the colony often appears to behave as a "superorganism" operating as a functionally integrated unit [3]. However, the concept of a super-organism as being the only unit at which natural selection operates has been challenged both on theoretical grounds and by the observation that life within the colony is not always as harmonious as it may first appear. Social life may involve conflicts of genetic self-interest, resulting in tactics of coercion, manipulation and even deadly aggression between colony members in the name of genetic self-interest. These conflicts arise because colony members may favor individuals that are more closely related (share more genes identical by common ancestry) to maximize their inclusive fitness [4]. These conflicts, in turn, have negative effects at the colony-level because they may decrease the overall productivity. These costs at the level of the colony are expected to lead to counter-strategies to suppress selfish behaviors [5]. In other words, the actual conflict should generally be lower than the potential conflict [6]. Understanding exactly how and to what degree actual conflict is suppressed and how this increases overall group productivity is the key to understanding the extent to which social insect colonies can be viewed as adaptively organized group-level units. In our ongoing project, we intend to investigate this issues by evolving colonies of artificial ants implemented as simulated robots endowed with simple behaviors. In order to ensure thorough statistical analysis, the evolutionary experiments have been carried out using genetic algorithms coupled to fast "minimalistic" simulations of the robotic evolutionary setup.

In this paper, we describe a set of four experiments, which have been used to test the effects of genetic relatedness and levels of selection in the evolution of cooperation and labor division. Relatedness is known to have played a major role in favoring the evolution of altruism in social insects $[7,8,5]$ and other animals and we would like to determine whether the role of relatedness can be experimentally demonstrated with artificial ants.

These explorations will provide hypotheses for the evolution of cooperation and division of labor in biological ants and guidelines for the design of autonomous robots capable of cooperation and task self-allocation, because most of the work done so far with real robots make use of pre-designed and fixed rules [9]. Accomplishing tasks with a multi-robot systems is appealing because of its analogous relationship with populations of social insects. Researchers argue that by organizing simple robots into cooperating teams, useful tasks may be accomplished otherwise impossible using a single robot [10].

In the next section we describe the model we have used to study the evolution of cooperation and the division of labor. In section 3 , we describe the set of experiments we have performed. In section 4 , we discuss our current results, and finally in section 5 , we present our concluding remarks. 


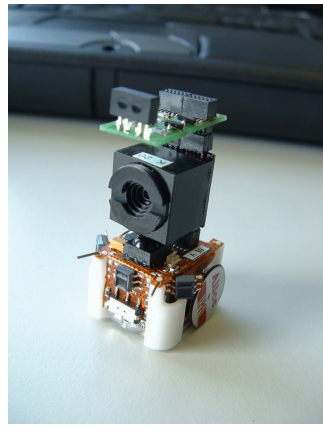

(a)

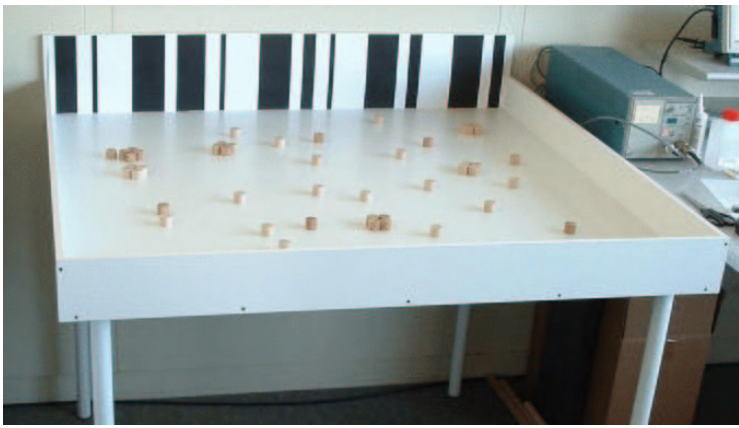

(b)

Fig. 1. Experimental setup. (a) Alice robot with proximity (infrared sensors), vision and radio modules. ALICE is a $21 \times 21 \times 20 \mathrm{~mm}$ micro-robot weighing less than $10 \mathrm{~g}$. It has an autonomy of up to 10 hours. (b) Real setup for the foraging task (the small and large objects represent food items, the wall with black and white stripes represents the nest).

\section{The model}

In theoretical biology, the study of the evolution of cooperation has been mainly undertaken using formal mathematical models and by using evolutionary game models [11]. A very well known example is the Prisoner's Dilemma game model [12] which has been particularly useful for studying the evolution of cooperation via reciprocity. The agent-based modeling approach is an alternative to the equationbased methods. These models offer the possibility to implement complex interactions between important parameters of the models which are sometimes impossible to incorporate into equation-based models, thus, the simplifications necessary to make the model tractable are less likely to bias the results [13].

\subsection{Foraging task}

We use an agent-based model of a colony of artificial ants performing a foraging task. The agents or artificial ants (e.g., robots or simulated robots) are supposed to look for food items randomly scattered in a foraging area. There are two kinds of food items, small food items which can be transported by single agents to the nest, and large food items, which can only be transported if two ants cooperate. When a cooperative foraging ant happens to find a large food item, it sends a local message asking for help. Given the local nature of the help message, another cooperative individual will only be able to help the first one, provided that it happens to be close to it and hear its message. For the sake of simplicity large food items can only be transported by a pair of ants and we have not included a pheromone-like communication among ants. 
In a preliminary set of experiments, we tested the technical feasibility of the experiments using an enhanced version of the ALICE micro-robot [14] including five infra-red sensors and a linear vision system (see Figure 1a). Interestingly, the ALICE micro-robot have limitations somehow similar to real ants due to their small size. First, the power available with the on-board batteries and the properties of the micro-motors limit the maximal force for pushing food items. Second, the complexity of the control software that can be implemented on the on-board micro-controllers is severely constrained by local-range sensors and relatively low computational power. Both these aspects, which can also be found in real ants, could benefit from cooperative strategies of a group of robots.

In Figure 1b) we show the real experimental setup where other members of our project tested the ALICE micro-robots and obtained robots capable of locating small objects (using infrared sensors) and pushing them toward a wall (i.e., the nest).

In the first phase of the project we use a "minimalistic" simulator to carry out a large number of experiments. The minimalistic simulator includes stochastic effects and time-dependent dynamics modeled upon the constraints of the physical setup.

In our experimental setup, each ant is endowed with a set of three 5 -bit genes $\left(g_{0}, g_{1}\right.$, and $\left.g_{2}\right)$ which encode three threshold values $\left(T_{0}, T_{1}\right.$, and $\left.T_{2}\right)$ that are used to determine if a given basic behavior $\left(b_{0}, b_{1}\right.$ or $\left.b_{2}\right)$ is activated or not during a step of a foraging trial.

\begin{tabular}{|c|c|c|}
\hline \multicolumn{3}{|r|}{\begin{tabular}{|l|l|l|l}
$b_{0}$ & $b_{1}$ & $b_{2}$ & Description of the resulting strategy \\
\end{tabular}} \\
\hline 0 & \begin{tabular}{|l|l}
0 & \\
\end{tabular} & \begin{tabular}{l|l}
0 & do nothing \\
\end{tabular} \\
\hline 1 & 0 & $\begin{array}{l}0 \text { if a small food item is found, bring it to the nest, ignore } \\
\text { large food items, and do not help other ants }\end{array}$ \\
\hline 0 & 1 & $\begin{array}{l}0 \text { if a large food item is found, stay and ask for help, ignore } \\
\text { small food items, and do not help other ants }\end{array}$ \\
\hline 0 & 0 & 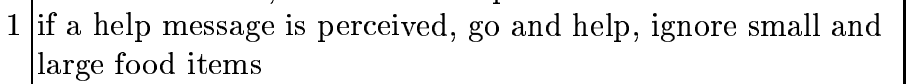 \\
\hline 1 & 1 & $\begin{array}{l}0 \text { if a small food item is found, bring it to the nest, if a large } \\
\text { food item is found ask for help, but do not help other ants }\end{array}$ \\
\hline 1 & 0 & $\begin{array}{l}1 \text { if a small food item is found, bring it to the nest, help } \\
\text { other ants, but ignore large food items }\end{array}$ \\
\hline 0 & 1 & 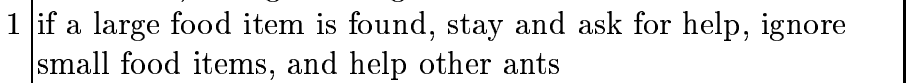 \\
\hline 1 & 1 & $\begin{array}{l}1 \text { if a small food item is found, bring it to the nest, if a large } \\
\text { food item is found, stay and ask for help, and help other ants }\end{array}$ \\
\hline
\end{tabular}

Table 1.

By varying the energetic value of the food items, one can put more or less pressure on the advantage of cooperative behaviors. For example, if a large food 
item is valued ten times more than a small one, it may pay off for ants to recruit other ants and transport the large food item toward the nest area even if this means that those ants will have to share it with the whole colony.

\subsection{Behavior activation}

The values of the binary variables $b_{0}, b_{1}$, and $b_{2}$ indicate whether a particular behavior, or a combination of behaviors has been activated or not (see Table 1). The members of a colony activate their behaviors in a random order.

The expression of a given behavior $b_{i}$ depends on the number of foragers already engaged in that behavior and is mediated by an individual threshold $T_{i}$ whose value is genetically encoded (i.e., by $g_{i}$ ). More precisely, if the proportion of members of the colony having activated a given behavior $j$ is smaller than the corresponding threshold $T_{j}^{k}$ of ant $k$, behavior $b_{j}^{k}$ is set to ' 1 ' (i.e., it is activated). This threshold mechanism was motivated by a model of division of labor in insect societies proposed by Bonabeau and colleagues [15]. In our model, high threshold values indicate that behaviors are more likely to be activated when several individuals have already activated that behavior than low threshold values, and low threshold values indicate that behaviors have less probability of being activated when several individuals have already activated that behavior.

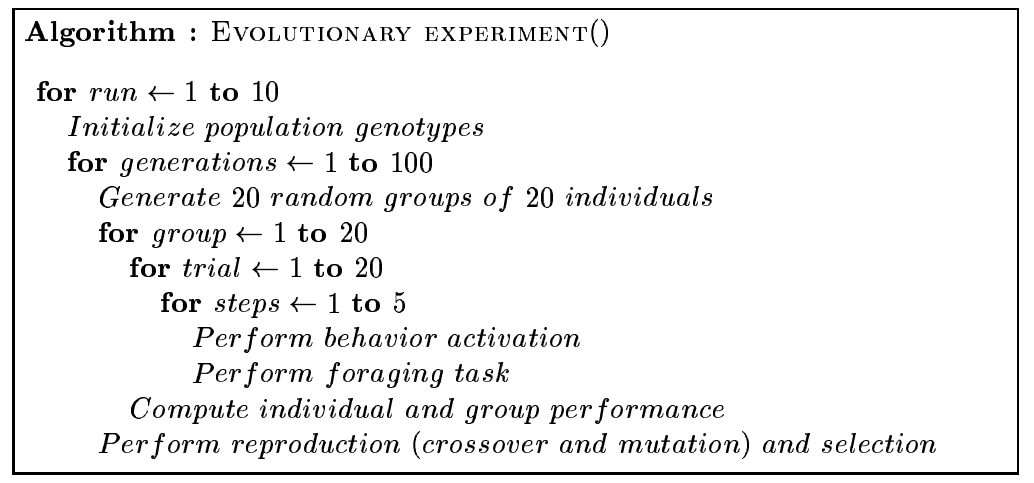

Fig. 2. General loop of an evolutionary experiment.

\subsection{Trial description}

A trial consists of a series of steps and every step is divided into two phases: in the first phase, each ant activates one or more basic behaviors (see section 2.2 ), while in the second phase, the group of 20 ants is tested on the foraging 


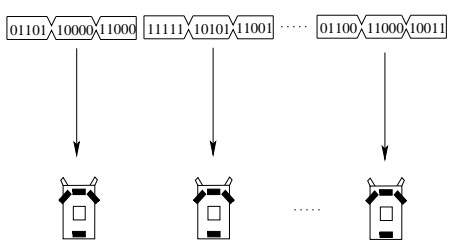

(a) Heterogeneous colonies

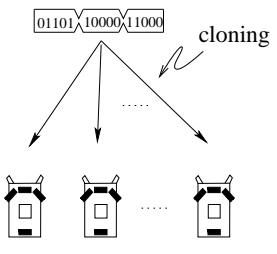

(b) Homogeneous colonies

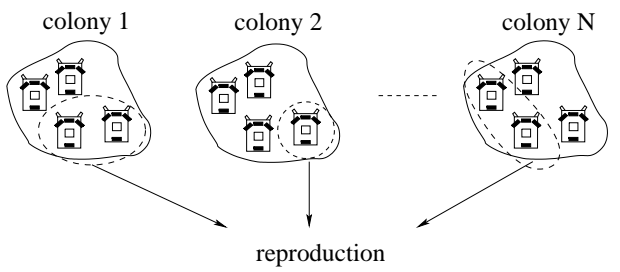

(c) Individual-level selection

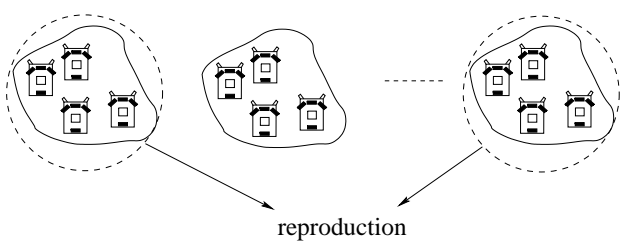

(d) Colony-level selection

Fig. 3. Generation of (a) heterogeneous and (b) homogeneous colonies, and implementation of (c) individual and (d) colony-level selection. See Section 3 for more details.

task. In the reported experiments there are 4 small and 4 large food items in the arena. To simulate the spatial distribution of the food items, an ant has a probability of $1-N O P P$ of finding a food item. In these experiments, the no-operation parameter NOPP was set to 0.2 , which means the ants happen to find an object with a probability of $80 \%$ during the first step of a trial. When the number of available food items diminishes (i.e., they are "transported to the nest") the probability for an ant to find an object also diminishes. Each colony has 5 time steps to achieve the foraging task. The performance of the colonies is then measured using the average score obtained during 20 different trials. In Figure 2 we show the whole sequence of steps of an experiment. See our web site (http://asl.epfl.ch) for a more detailed description of the "minimalist" probabilistic simulator of the foraging task.

In our experiments, the small food items provided a score of 1.0 to the single ant having transported it to the nest, while the large food items provided a total score of 16.0. However, the large food items are shared with the whole colony, thus each individual gets a score of 0.8 when a couple of cooperating ants "managed to transport" a large food item to the nest. According to these payoffs, the individuals that do not cooperate can get 0.8 points for every large food item transported by other individuals of the colony, whereas the individuals that cooperate and share food, have a cost of 0.2 points compared to the score they would made if they concentrated on the small food items. Nevertheless, the total performance of the colony is maximized if the individuals prefer to transport large food items than acting "selfishly". 


\section{Experiments}

Four experiments have been designed to determine to what extent the level of cooperation among ants is influenced by the level of genetic relatedness (heterogeneous or homogeneous colonies) and the level of selection: individual or colony-level selection (See Figure 3). The four experiments have been carried out using a form of artificial evolution where only the "fittest" individuals or colonies are allowed to reproduce [16]. The offspring are generated by exchanging genes between the parents with a probability of $20 \%$ (crossover probability) and by adding a random value to each gene.

A population of 400 individuals is initialized by setting up their genotypes as follows: $g_{0}=\operatorname{rnd}\left(' 00000\right.$ ', '11111'), that is, a 5-bit random string, $g_{1}=$ '00000', and $g_{2}=$ '00000'. This means that the whole population is initially composed of individuals that do not cooperate. The individuals are organized into 20 colonies of 20 ants each. Individuals of a colony can be all clones (homogeneous) or have different genes (heterogeneous). In the case of individual-level selection, individuals are selected from different colonies on the basis of their performance, and reproduced to form 20 new colonies. In the case of colony-level selection, all individuals of the colonies with the highest fitness are reproduced to form 20 new colonies.

\section{Results and discussion}

In Figure 4 we show the evolution (during 100 generations) of the frequency of altruistic individuals in the simulated ant populations according to the four experimental setups, previously described. Herein, we consider an individual to be "altruistic" when it does not "pay attention" to the small food items and concentrate only on the large food items, whether looking for large food items or helping other individuals to transport large food items.

Figure 4 shows the evolution of the mean frequency of individuals in the population having activated the strategies: $\left[b_{0}=0, b_{1}=1, b_{2}=0\right],\left[b_{0}=0, b_{1}=\right.$ $\left.0, b_{2}=1\right]$, and $\left[b_{0}=0, b_{1}=1, b_{2}=1\right]$. Notice that the percentage of altruistic individuals within the population of heterogeneous colonies evolved using individual-level selection remains below $10 \%$. However, in the other three setups there is a gradual dominance of altruistic individuals in the population. In particular, the resulting number of altruistic individuals is higher when using colony-level selection (Figure $4 \mathrm{~b}$ and Figure $4 \mathrm{~d}$ ). This is understandable because colony-level selection favors the individuals that work for the colony and not the ones that specialize in the foraging of small food items for their own benefit.

In Figure 5 we show the evolution of the mean performance of the homogeneous and heterogeneous colonies evolved using individual and colony-level selection. The performance differences appear to be related to genetic relatedness. Indeed, the homogeneous colonies display higher mean fitness than the heterogeneous colonies after the 100-generation runs. In order to validate this, we performed 10-run evolutionary experiments during 2000 generations and measured the mean performance from generation 1000 to 2000 . Then, we performed 


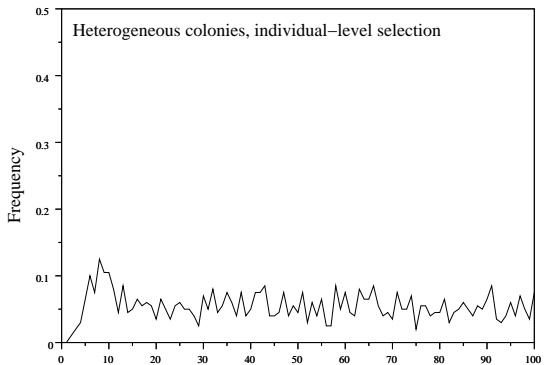

(a)

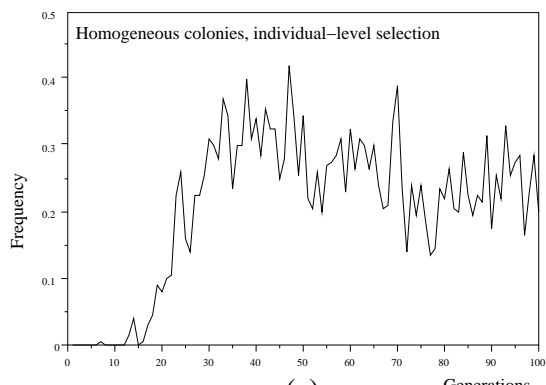

(c)

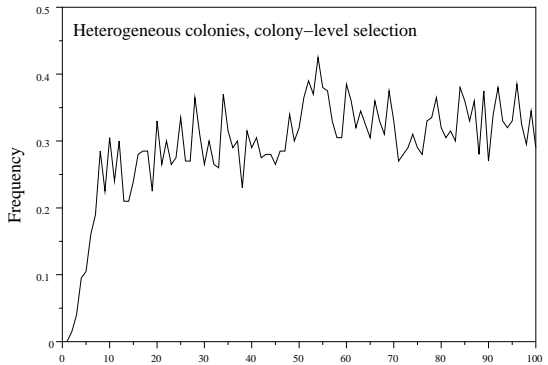

(b)

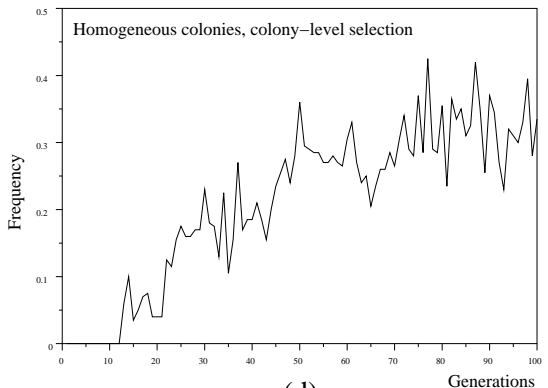

(d)

Fig. 4. Evolution of the frequency of altruistic individuals in the simulated ant populations (average of 10 runs) given the following experimental setups: (a) Heterogeneous colonies, individual-level selection, (b) Heterogeneous colonies, colony-level selection, (c) Homogeneous colonies, individual-level selection, and (d) Homogeneous colonies, colony-level selection.

a Wilcoxon test to determine if the difference between the mean performance of the colonies were "statistically significant". These results show that: (1) the mean performance of the homogeneous colonies evolved using colony-level selection $P_{H m C}$ is higher than the mean performance of the heterogeneous colonies evolved using colony-level selection $P_{H t C}$, that is, $P_{H m C}>P_{H t C}[p<0.0001]$; (2) the mean performance of the homogeneous colonies evolved using individuallevel selection $P_{H m I}$ is higher than the mean performance of the heterogeneous colonies evolved using individual-level selection $P_{H t I}$, that is, $P_{H m I}>P_{H t I}$ $[p<0.0001]$; (3) the mean performance of the heterogeneous colonies evolved using colony-level selection $P_{H t C}$ is higher than the mean performance of the heterogeneous colonies evolved using individual-level selection $P_{H t I}$, that is, $P_{H t C}>P_{H t I}[p<0.0001]$. However, there is no significant difference between the mean performance of the homogeneous colonies evolved using colony-level selection $P_{H m C}$ and the mean performance of the homogeneous colonies evolved using individual-level selection $P_{H m I}$. 


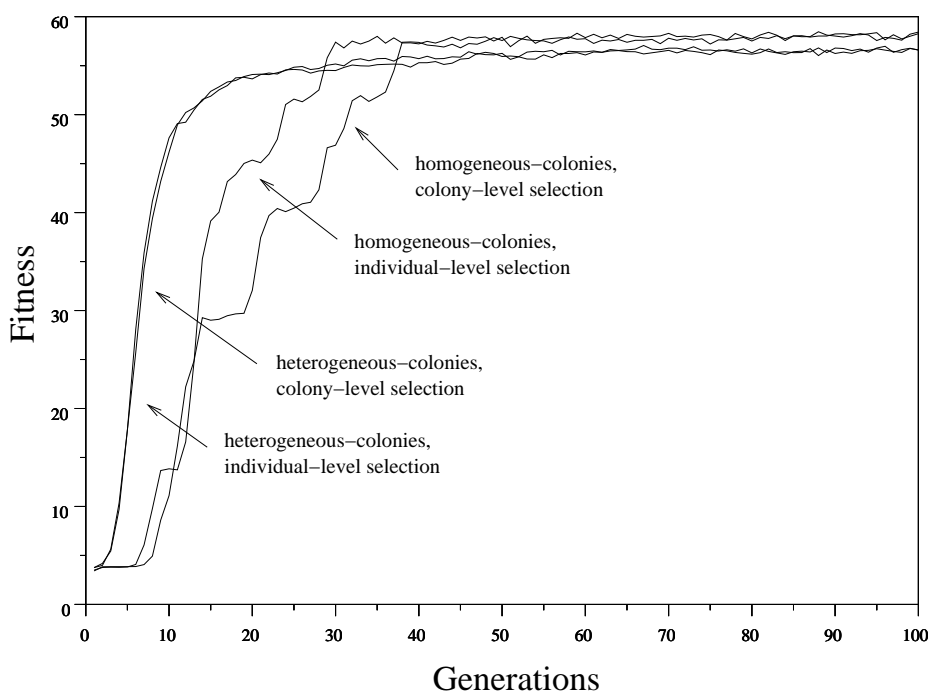

Fig. 5. Evolution of the mean performance of homogeneous and heterogeneous colonies under individual and colony-level selection (each curve is the average of 10 different runs).

\section{Concluding remarks}

In this paper we present a set of experiments, where minimalistic simulations are used to study the effects of group composition and levels of selection in the evolution of cooperation in artificial ants. (1) We show that altruistic behaviors are favored by colony-level selection and that altruistic behaviors have low probability of emerging in heterogeneous colonies evolving under individual-level selection. (2) Concerning the prediction, made by some biologists $[17,18]$, that groups should be more efficient when selection acts at the level of the colony and when there is higher relatedness within groups, we have so far found that homogeneous colonies, indeed, performed better than heterogeneous ones. However, we did not find a clear superiority in the performance of colonies evolved using colony-level selection. This may be due to the fact that individuals can simultaneously activate self-interest and altruistic behaviors. We are currently pursuing our research performing further exploration of these issues and the evolution of division of labor. Our next step is to validate these experiments with the physical robots, where interaction dynamics may significantly affect costs and benefits. 


\section{Acknowledgments}

The authors gratefully acknowledge the support from the Fonds UNIL-EPFL 2002, the Rectorate of the University of Lausanne, and the Swiss National Science Foundation. Moreover, we thank A. Colot and G. Caprari for their help with the ALICE robot, the implementation of the real arena, the development and test of the new ALICE robots, and the pictures.

\section{References}

1. Hölldobler, B., Wilson, E.: The Ants. Springer-Verlag, Berlin (1990)

2. Gadagkar, R.: Survival strategies: cooperation and conflict in animal societies. Harvard University Press (1997)

3. Wheeler, W.: The social insects: their origin and evolution. Kegan Paul, London (1928)

4. Hamilton, W.: The genetical evolution of social behavior 2. Journal of Theoretical Biology 7 (1964) 17-52

5. Keller, L., Reeve, H.: Dynamics of conflicts within insect societies. In: Levels of Selection in Evolution. Princeton University Press (1999) 153-175

6. Ratnieks, F., Reeve, H.: Conflict in single-queen hymenopteran societies: the structure of conflict and processes that reduce conflict in advanced eusocial species. Journal of Theoretical Biology 158 (1992) 33-65

7. Bourke, A., Franks, N.: Social evolution in ants. Princeton University Press (1995)

8. Sundström, L., Chapuisat, M., Keller, L.: Manipulation of sex ratios by ant workers: A test of kin selection theory. Science 274 (1996) 993-995

9. Krieger, M., Billeter, J., Keller, L.: Ant-like task allocation and recruitment in co-operative robots. Nature 406 (2000) 992-995

10. Brooks, R.: Challenges for complete creature architectures. In Meyer, J.A., Wilson, S., eds.: From animals to animats. First International Conference on Simulation of Adaptive Behavior, The MIT Press (1991) 434-443

11. Dugatkin, L., Reeve, H., eds.: Game Theory and Animal Behavior. Oxford University Press (1998)

12. Axelrod, R., Hamilton, W.: The evolution of cooperation. Science 211 (1981) 1390-1396

13. Pepper, J., Smuts, B.: The evolution of cooperation in an ecological context: an agent-based model. In Kohler, T.A., Gumerman, G.J., eds.: Dynamics in Human and Primate Societies: Agent-Based Modeling of Social and Spatial Processes, Oxford University Press (2000) 45-76

14. Caprari, G., Estier, T., Siegwart, R.: Fascination of down scaling - alice the sugar cube robot. Journal of Micro-Mechatronics 1 (2002) 177-189

15. Bonabeau, E., Theraulaz, G., Schatz, B., Deneubourg, J.L.: Response threshold model of division of labour in a ponerine ant. Proc. Roy. Soc. London B 265 (1998) $327-335$

16. Nolfi, S., Floreano, D.: Evolutionary Robotics. The Biology, Intelligence, and Technology of Self-organizing Machines. The MIT Press, Cambridge, Massachusetts (1999)

17. Keller, L., Ross, K.: Selfish genes: a green beard in the red fire ant. Nature 394 (1998) 573-575

18. Keller, L., Chapuisat, M.: Cooperation among selfish individuals in insect colonies. BioScience 49 (1999) 899-909 